\title{
Two-channel approach to the average retarding force of metals for slow singly ionized projectiles
}

\author{
I. Nagy ${ }^{1,2}$ and I. Aldazabal $\oplus^{3,2}$ \\ ${ }^{1}$ Department of Theoretical Physics, Institute of Physics, Budapest University of Technology and Economics, 1521 Budapest, Hungary \\ ${ }^{2}$ Donostia International Physics Center, P. Manuel de Lardizabal 4, 20018 San Sebastián, Spain \\ ${ }^{3}$ Centro de Física de Materiales, CSIC-UPV/EHU, P. Manuel de Lardizabal 5, 20018 San Sebastián, Spain
}

(Received 8 June 2020; accepted 27 August 2020; published 15 September 2020)

\begin{abstract}
Based on the fundamental momentum-transfer theorem [B. A. Lippmann, Phys. Rev. Lett. 15, 11 (1965)], a contribution to the retarding force of metallic systems for slow intruders is derived. This contribution is associated with sudden charge-changing cycles during the path of projectiles. The sum of this and the well-known conventional contributions, both expressed in terms of scattering phase shifts, is used to discuss experimental data obtained for different targets. It is found that our two-channel modeling, with two nonlinear channels, improves the agreement between data and theory and thus, as predictive modeling, can contribute to the desired convergence between experimental and theoretical attempts at the retarding force.
\end{abstract}

DOI: 10.1103/PhysRevA.102.032814

\section{INTRODUCTION AND MOTIVATION}

According to a basic book on quantum mechanics by Landau and Lifshitz [1], one of the most important quantities in the interaction of heavy charged projectiles with fixed atoms is the average energy loss. This time-independent quantity, a kind of deposited energy, is an observable and due to conservation laws its measurement is feasible in experiments. Thus, in this subfield of nature (physics and human therapy) the real challenge resides in the convergence of measurements and theories. Their interplay, a continuous one over a century, fertilizes the development of both approaches, which can result in transferable knowledge [2].

The present work is dedicated to the case where singly ionized projectiles interact with constituents of metallic targets. The main challenge addressed here is to find a reasonable combination of the quantum-statistical and atomistic aspects of the energy-loss process in real targets. As motivation, on which our attempt is partially based, we start with an established result. The well-known conventional form [3-5] for the stopping power (written in Hartree atomic units, where $e^{2}=m_{e}=\hbar=1$ ) of a homogeneous degenerate electron gas (characterized by Fermi velocity $v_{F}$ ) for heavy intruders is given by

$$
\frac{d E}{d x}=\frac{2}{(2 \pi)^{3}} \int_{0}^{v_{F}} v_{e}^{2}\left[2 \pi \int_{-1}^{1} d x\left(v-v_{e} x\right) v_{r} \sigma_{\mathrm{tr}}\left(v_{r}\right)\right] d v_{e},
$$

where $v$ and $v_{e} \in\left[0, v_{F}\right]$ are the projectile and systemelectron velocities. One of the integration variables is $x \equiv$ $\cos \beta$, where $\beta$ is the angle between $\mathbf{v}$ and $\mathbf{v}_{e}$. Thus $v_{r}^{2}=v^{2}+v_{e}^{2}-2 v_{e} v x$. Clearly, in an interpretation based on independent-electron scattering off a heavy projectile moving with constant velocity $v$, the remaining task resides in a common two-body interaction $V(r)$, in order to perform the statistical averaging over a Fermi-Dirac distribution with

$$
\sigma_{\mathrm{tr}}\left(v_{r}\right)=\frac{4 \pi}{v_{r}^{2}} \sum_{l=0}^{\infty}(l+1) \sin ^{2}\left[\delta_{l}\left(v_{r}\right)-\delta_{l+1}\left(v_{r}\right)\right] .
$$

In this scattering interpretation the analysis is based on the concept of asymptotic states in the infinite past and future, i.e., involving large time differences. Sudden processes in time, like a local charge change in metals, require a refined approach on associated transition amplitudes in time-dependent perturbation theory. In kicklike sudden [1] processes one may use predetermined states as a complete set to treat the matrix elements in strong (but transient in time) perturbations. Note that it is precisely such a transient channel which could create difficulties in large-scale simulations, like in the orbital-based implementation of time-dependent density-functional theory (TDDFT).

Such an implementation rests on averaging of quantummechanical time-dependent energy differences over certain timescales in order to define a forcelike quantity such as a stationary observable in stopping [6,7]. In these large-scale simulations specification of the initial conditions is required for real-time propagation. For instance, in [7] two options were considered for helium in aluminum target. In the first one, the screened atom was included in the determination of the static initial state. In the second one, the initial condition was set up by adding an $\alpha$ particle and thus producing a sudden change in the external potential. In both cases the authors control only the initial state and not the subsequent dynamics which is given by the time-dependent single-particle equations within TDDFT. Therefore, a smoothed evolving picture, without fast local charge-changing processes, is employed.

First, as concretization of our motivation, we integrate Eq. (1) by using models for the momentum-transfer cross section in order to get useful information about the phenomenology discussed in Sec. II after Eq. (6). Namely, we 
take the form of $\sigma_{\mathrm{tr}}\left(v_{r}\right)=4 \pi A_{\alpha} / v_{r}^{\alpha}$, in which $\alpha=2$ and 4 . By straightforward quadrature we obtain [5] from Eq. (1) for these models

$$
\begin{aligned}
\frac{d E}{d x} & =\frac{4 \pi A_{2}}{\left(v_{F}\right)^{2}} n_{0} v v_{F}\left[1-\frac{1}{5}\left(\frac{v}{v_{F}}\right)^{2}\right] \\
& =A_{2} \frac{4}{3 \pi} v_{F}^{2} v\left[1-\frac{1}{5}\left(\frac{v}{v_{F}}\right)^{2}\right] \quad \text { for } v \leqslant v_{F}, \\
\frac{d E}{d x} & =\frac{4 \pi A_{2}}{(v)^{2}} n_{0} v^{2}\left[1-\frac{1}{5}\left(\frac{v_{F}}{v}\right)^{2}\right] \quad \text { for } v \geqslant v_{F}, \\
\frac{d E}{d x} & =\frac{4 \pi A_{4}}{\left(v_{F}\right)^{4}} n_{0} v v_{F}=A_{4} \frac{4}{3 \pi} v \quad \text { for } v \leqslant v_{F}, \\
\frac{d E}{d x} & =\frac{4 \pi A_{4}}{(v)^{4}} n_{0} v^{2} \quad \text { for } v \geqslant v_{F} .
\end{aligned}
$$

The above model results are of course in agreement with the expected limits $d E / d x=n_{0} v v_{F} \sigma_{\mathrm{tr}}\left(v_{F}\right)$ and $d E / d x=$ $n_{0} v^{2} \sigma_{\mathrm{tr}}(v)$ at $v \rightarrow 0$ and $v \rightarrow \infty$, respectively. Earlier, careful theoretical analysis [8] performed within an adiabatic framework on velocity dependence stated that the next term beyond the $v$-proportional one is at least second order in velocity. Our closed expressions for $v \leqslant v_{F}$ are in harmony with this important statement. Furthermore, a certain weighted combination of our two expressions at $v \leqslant v_{F}$ would result in an almost perfect $v$ proportionality. That, at this point, simple mathematical observation will become a more transparent and physical one in Sec. II, where we extend the theory on the average retarding force beyond the common fixed-potential approximation by considering physically reasonable force matrix elements as independent channel contributions.

The rest of this paper is organized as follows. Section II is devoted to theory and a discussion of the results obtained. Section III contains a short summary and a few general comments. As above, we use atomic units throughout this work.

\section{RESULTS AND DISCUSSION}

We begin this section by outlining few important elements of stationary scattering theory. According to the basic rules of quantum mechanics on expectation values of operators, one should consider the force matrix element [9] between orthonormal components of a scattering state to characterize the associated momentum transfer. Applying this quantummechanical theorem, where $\sigma_{\mathrm{tr}}\left(v_{r}\right) \propto \sum_{l=0}^{\infty}(l+1)\left[I_{1}\left(l, v_{r}\right)\right]^{2}$, one has [10-12] for the matrix elements

$$
\begin{aligned}
I_{1}\left(l, v_{r}\right) & =\left[\int_{0}^{\infty} d r r^{2} R_{l}\left(r, v_{r}\right) \frac{\partial V(r)}{\partial r} R_{l+1}\left(r, v_{r}\right)\right] \\
& =\sin \left[\delta_{l}\left(v_{r}\right)-\delta_{l+1}\left(v_{r}\right)\right] .
\end{aligned}
$$

We stress that this remarkable identity rests on those states characterized by the scattering Schrödinger wave equation with $v_{r}^{2} / 2$ energy and $V(r)$ external field. However, with partial waves based on $V(r)$, but with a net Coulomb field
$\Delta V_{c}(r)=-q / r$ in space of $V(r)$, we get

$$
\begin{aligned}
I_{2}\left(l, v_{r}\right) & =\left[\int_{0}^{\infty} d r r^{2} R_{l}\left(r, v_{r}\right) \frac{\partial}{\partial r}\left(-\frac{q}{r}\right) R_{l+1}\left(r, v_{r}\right)\right] \\
& =\frac{q \cos \left[\delta_{l}\left(v_{r}\right)-\delta_{l+1}\left(v_{r}\right)\right]}{2 v_{r}(l+1)},
\end{aligned}
$$

and with unperturbed $(u)$ partial-wave components the corresponding result becomes

$$
\begin{aligned}
I_{2}^{(u)}\left(l, v_{r}\right) & =\left[\int_{0}^{\infty} d r r^{2} j_{l}\left(v_{r} r\right) \frac{\partial}{\partial r}\left(-\frac{q}{r}\right) j_{l+1}\left(v_{r} r\right)\right] \\
& =\frac{q}{2 v_{r}(l+1)} .
\end{aligned}
$$

Here we used the spherical Bessel functions of the first kind, i.e., the components of an unperturbed plane-wave (momentum) state, instead of self-consistent radial functions. These forms in Eqs. (4) and (5) are based on the fact that in cases with abrupt perturbations the original stationary system has no time [1] to relax to the stationary state of a new Hamiltonian.

We will consider these amplitudes as the proper ones when there is a sudden change in the self-consistent $V(r)$, as in the case of charge-changing $(q=1)$ processes generated by the binary interaction with fixed lattice ions. This charge change results in an excess bare field $\Delta V_{c}(r)=-1 / r$. The square of $I_{2}\left(l, v_{r}\right)-I_{2}^{(u)}\left(l, v_{r}\right)$ can characterize, in a quantum-mechanical interpretation, an extra (kicklike) momentum transfer due to the sudden change in the external field. That square is in fact a regularized transition probability. Such a regularization is needed since both $I_{2}$ and $I_{2}^{(u)}$ would give divergent results after $l$ summation. This regularized channel gives (at $q \neq 0$ ) a form for the associated cross section

$$
\sigma_{\mathrm{tr}}^{(2)}\left(v_{r}\right)=\frac{4 \pi}{v_{r}^{2}}\left(\frac{q}{v_{r}}\right)^{2} \sum_{l=0}^{\infty} \frac{1}{l+1} \sin ^{4}\left[\frac{\delta_{l}\left(v_{r}\right)-\delta_{l+1}\left(v_{r}\right)}{2}\right],
$$

for which a simple trigonometrical identity $(1-\cos \alpha)^{2}=$ $4 \sin ^{4}(\alpha / 2)$ is employed.

Before our quantitative analysis, we continue with phenomenology. There are important differences between Eqs. (6) and (2), i.e., between $\sigma_{\mathrm{tr}}^{(2)}\left(v_{r}\right)$ and the conventional one given by Eq. (2) and denoted from here by $\sigma_{\text {tr }}^{(1)}\left(v_{r}\right)$. The kinematical prefactors show that the new term (proportional to $v_{r}^{-4}$ ) vanishes faster at large scattering wave number $v_{r}$. Thus, at $v_{r} \leqslant 1$ values, which represent, at a small intruder velocity, the range of the Fermi velocity of metals, and at $\delta \simeq \pi$ for a dominating phase shift, the new term can become the dominating one. A combination of the $v_{r}^{-2}$ and $v_{r}^{-4}$ dependences in $\sigma_{\mathrm{tr}}^{(1)}\left(v_{r}\right)+\sigma_{\mathrm{tr}}^{(2)}\left(v_{r}\right)$ with the integrated characteristics found with separated model cross sections in the Introduction signals that a velocity proportionality in the stopping power holds, practically up to $v \simeq v_{F}$ from below.

Now we turn to the quantitative part of this section. We will determine numerically the two quantities, denoted by $Q^{(1)}\left(v_{F}\right)$ and $Q^{(2)}\left(v_{F}\right)$, by which the low-velocity stopping power of metals (a system of an electron gas and lattice ions) takes a frictionlike form

$$
\frac{1}{v} \frac{d E}{d x}=Q^{(1)}\left(v_{F}\right)+Q^{(2)}\left(v_{F}\right)
$$


where the two coefficients (when $q \neq 0$ ) are given by the expressions

$$
\begin{aligned}
& Q^{(1)}\left(v_{F}\right)=\frac{4}{3 \pi} v_{F}^{2} \sum_{l=0}^{\infty}(l+1) \sin ^{2}\left[\delta_{l}\left(v_{F}\right)-\delta_{l+1}\left(v_{F}\right)\right] \\
& Q^{(2)}\left(v_{F}\right)=\frac{4}{3 \pi} q^{2} \sum_{l=0}^{\infty} \frac{1}{l+1} \sin ^{4}\left[\frac{\delta_{l}\left(v_{F}\right)-\delta_{l+1}\left(v_{F}\right)}{2}\right] .
\end{aligned}
$$

Our summation of two-channel cross sections in Eq. (7) resembles, mathematically, the well-known [13] rule in potential scattering with a spin-orbit interaction term where we sum the direct (non-spin-flip) and spin-flip partial differential cross sections for electron scattering for any spin orientation before scattering. There the integrated cross sections, needed for observables, are obtained by integrating over all scattering angles. Remarkably, the spin-flip part depends on an amplitude difference, similar to our regularized difference.

We stress at this point that we employ in the summation in Eq. (7) an a priori unit-weight assumption. In reality, i.e., for channelinglike conditions in metals, the impact parameterdependent closest approach of intruders and lattice ions [7,1416] may influence that assumption. In more simple terms, our present weighting would refer to random-collision situations. Nonequal weighting might be based on certain probabilistic inputs [17] to sum two nonlinear channel; however, such inputs need, in our modeling, an additional justification, since one cannot apply stationary linear-response ideas to a sudden effect.

Table I contains our numerical results for $Q^{(1)}\left(v_{F}\right)$ and $Q^{(2)}\left(v_{F}\right)$ at selected values of the $r_{s}$ Wigner-Seitz radius and at $q=1$. The partial phase shifts, calculated by DFT at the Fermi momentum, are taken from earlier works [18-20]. Both $Q^{(1)}$ and $Q^{(2)}$ are oscillating functions, but $Q^{(1)}\left(r_{s}\right)$ has a strong

TABLE I. Partial contributions $Q^{(1)}\left(v_{F}\right)$ and $Q^{(2)}\left(v_{F}\right)$ at $q=1$ to Eq. (7). Phase shifts, based on the orbital version of DFT [18-20],

\begin{tabular}{|c|c|c|c|c|c|c|}
\hline \multirow[b]{2}{*}{$Z_{1}$} & \multicolumn{2}{|c|}{$r_{s}=1.5$} & \multicolumn{2}{|c|}{$r_{s}=2$} & \multicolumn{2}{|c|}{$r_{s}=3$} \\
\hline & $Q^{(1)}$ & $Q^{(2)}$ & $Q^{(1)}$ & $Q^{(2)}$ & $Q^{(1)}$ & $Q^{(2)}$ \\
\hline 1 & 0.305 & 0 & 0.255 & 0 & 0.162 & 0 \\
\hline 2 & 0.755 & 0.069 & 0.427 & 0.134 & 0.135 & 0.250 \\
\hline 3 & 0.912 & 0.161 & 0.439 & 0.247 & 0.117 & 0.368 \\
\hline 4 & 1.112 & 0.235 & 0.557 & 0.323 & 0.191 & 0.421 \\
\hline 5 & 1.417 & 0.298 & 0.749 & 0.374 & 0.307 & 0.443 \\
\hline 6 & 1.692 & 0.366 & 0.874 & 0.413 & 0.346 & 0.481 \\
\hline 7 & 1.777 & 0.369 & 0.825 & 0.449 & 0.275 & 0.522 \\
\hline 8 & 1.631 & 0.402 & 0.637 & 0.483 & 0.167 & 0.545 \\
\hline 9 & 1.346 & 0.438 & 0.428 & 0.512 & 0.085 & 0.563 \\
\hline 10 & 1.047 & 0.471 & 0.267 & 0.539 & 0.035 & 0.593 \\
\hline 11 & 0.815 & 0.498 & 0.183 & 0.564 & 0.032 & 0.612 \\
\hline 12 & 0.690 & 0.520 & 0.199 & 0.572 & 0.108 & 0.595 \\
\hline 13 & 0.697 & 0.531 & 0.338 & 0.559 & 0.242 & 0.546 \\
\hline 14 & 0.850 & 0.533 & 0.580 & 0.527 & 0.348 & 0.484 \\
\hline 15 & 1.146 & 0.521 & 0.846 & 0.482 & 0.360 & 0.427 \\
\hline 16 & 1.539 & 0.502 & 1.062 & 0.437 & 0.297 & 0.405 \\
\hline 17 & 1.975 & 0.480 & 1.219 & 0.404 & 0.234 & 0.374 \\
\hline 18 & 2.386 & 0.458 & 1.364 & 0.386 & 0.191 & 0.372 \\
\hline
\end{tabular}
are employed. See the text for further details. direct density dependence via $v_{F}^{2} \propto r_{s}^{-2}$ in Eq. (8). Thus, at metallic densities, $Q^{(2)}\left(r_{s}\right)$ in the sum $Q^{(1)}+Q^{(2)}$ can make an important modulation in the $Z_{1}$ oscillation of $Q^{(1)}$, especially around its minima. For $Z_{1}=1$, we take our values for $Q^{(1)}$ obtained within the explicit version [5] of DFT. There a single Euler equation is solved in an iterative self-consistent way. That calculation does not consider a doubly populated weakly bound (extended) state around an embedded proton in an electron gas, in harmony with experimental facts, obtained by positive muons, on the nonexistence of muonium in metals.

Despite this, there is perfect agreement [5] with $Q^{(1)}$ results obtained from the implicit orbital-based DFT. This agreement signals that it is the short-range part of proton screening which needs a nonlinear treatment. In simple terms, that range is the most important one to determine the first few phase shifts. Our $Q^{(2)}=0$ values for $Z_{1}=1$ are in accord with a screened-proton picture without a bound state, where there is no charge-changing cycle; thus $q=0$ during the motion of the projectile. Since the experimental data, obtained with low-velocity proton projectiles for $\mathrm{Al}$ and $\mathrm{Ni}$ targets, are in reasonable harmony $[15,16,21]$ with nonlinear theory [18-20] based solely on $Q^{(1)}\left(v_{F}\right)$, we have transferable knowledge in this case. A desired convergence between the two sides of understanding is achieved.

For all other $Z_{1} \geqslant 2$ we consider, for velocities $v \leqslant v_{F}$, the $q=1$ value as the most plausible one. This conservative value seems to be a realistic one with singly ionized intruders. Higher- $q$ values might have relevance when there is a large electronic overlap between clouds of colliding atoms. We believe that such a partial channel with $q>1$ would need more-energetic head-on-like collisions. Theoretically, it would be interesting to model the transition from our discrete$q$ modeling of charge-changing cycles to the pioneering [22] quasiclassical work where the retarding force (the observable) is related to an electron density flux constructed from the statistical Thomas-Fermi theory of atoms. With a transition study one might arrive at a deeper understanding of an integrated (classical trajectory Monte Carlo) approach [23] for energy-loss and -capture processes.

There is certain contradiction (cf. Fig. 2 below) for the $\mathrm{Al}$ target between the low-velocity experiment [15] and TDDFT $[6,7,24]$ results in the case of $\mathrm{He}^{+}$projectiles. In this case our result, based on $Q^{(1)}+Q^{(2)}$, is in harmony with [7] for the off-channeling situation. For the channeling simulation our $Q^{(1)}$ also gives reasonable agreement with [6,7]. We stress that [24] uses an $\alpha$ particle $\left(\mathrm{He}^{2+}\right)$ as the projectile and an atom-centered optimized Gaussian basis set to model the energy transfer. The observed agreement (see the discussion around Fig. 2) is remarkable in light of the careful experiment [25] performed on electron emission from aluminum. There perfect linearity in the velocity of helium ions, with $v \leqslant 0.6$, was obtained, and thus quantitative agreement with [6] was concluded.

Note, in the spirit of the discussion made already in [7] for proton and helium intruders, that we can image an experimental situation where, at very low ion velocities, only the neutral screened atom contributes via its $Q^{(1)}$, and the $Q^{(2)}$ channel becomes active only from an intermediate velocity below the Fermi velocity. Such a modeling would fit the experimental [15] suggestion on two (both linear in ion velocity) parts on 
the whole kinematical range $v \leqslant v_{F}$. Of course, the acceptance of such a suggestion presupposes that the underlying experiment-evaluation method behind the data is well justified for the whole range of ion velocity. For these simple intruders, partial support of such a view could be based on the surfacescattering experiment [26] performed with singly ionized ions $\left(Z_{1} \in[1,20]\right.$ and at projectile velocity $\left.v=0.5\right)$ scattered from an aluminum surface at variable scattering angles. There the challenging problem of inhomogeneity in the electron density profile of the electron salvage in front of a metal surface was studied, along with the phenomenological refinement of factors in $d E / d x=\left(n_{0} v\right)\left[v_{F} \sigma_{\mathrm{tr}}^{(1)}\left(v_{F}\right)\right]$. We will return to this experiment in the discussion of Fig. 3, which is devoted to an important comparison for $Z_{1}>2$.

Related to our prediction with $Q^{(1)}+Q^{(2)}>Q^{(1)}$ values for the average retarding force in metals, we turn to a brief discussion of data [14] obtained for another free-electron-like material, $\mathrm{Mg}$. For this metal $v_{F} \simeq 0.7$ and the experiment with $\mathrm{He}^{+}$and proton projectiles was performed for $v \geqslant v_{F}$. It was found that the ratio $R$ of stopping powers with these intruders becomes about 2 , in contrast to a ratio of about unity which is based on $Q^{(1)}$ values of self-consistent DFT. Our approach would result in a ratio $(R>2)$ which is not in contradiction with experimental suggestion. As support, we note that in [26], i.e., in a surface experiment, the helium per proton stopping ratio was found to be always higher than unity, even for $r_{s}(z)>3$. Clearly, the desired convergence of theories and experiments requires further studies for $\mathrm{Mg}\left(r_{s} \simeq 2.7\right)$ and, say, for $\mathrm{Ca}\left(r_{s} \simeq 3\right)$ as well, within large-scale TDDFT simulations with proton and helium intruders at $v \leqslant v_{F}$.

At this point, i.e., before the presentation and discussion of our illustrative figures, we would like to mention a very recent attempt [27] where a two-channel modeling was presented for the spectral linewidth in plasma environments. The authors of that insightful work demonstrated that the commonly used expression for the linewidth neglects a potentially important contribution from electron-capture processes. Their numerical value signals that a proper sum of two contributions can be about twice the conventional estimation. In the field of high-energy-density plasmas, our $q$-mediated enhancement in stopping power may contribute to the proper determination of the ignition threshold [28] in a deuterium-tritium- $\alpha$ energydeposition process. There, via a plausible postulation, the theoretical underprediction of stopping data was associated [28] with ion-ion nuclear scattering.

Now we illustrate our results with three figures. In Fig. 1, for $r_{s}=1.5$ of the Wigner-Seitz parameter, we plot the dimensionless ratios of $R_{1}=\left[Q^{(1)}\left(v_{F}, Z_{1}\right) / Q^{(1)}\left(v_{F}, Z_{1}=\right.\right.$ $1)]^{1 / 2}$ and $R_{2}=\left\{\left[Q^{(1)}\left(v_{F}, Z_{1}\right)+Q^{(2)}\left(v_{F}, Z_{1}\right)\right] / Q^{(1)}\left(v_{F}, Z_{1}=\right.\right.$ $1)\}^{1 / 2}$, i.e., ratios of nonlinear quantities. One might consider [21,29] these ratios as a kind of effective charge. This figure reflects, in a highly phenomenological manner, that the so-called $Z_{1}$ oscillations may get important modulations especially around the minima of the conventional $R_{1}$ ratio. Notice that the ratios so defined are square roots of physical magnitudes. This mathematical operation has a smoothing character (cf. Fig. 3) with renormalized oscillating functions.

Figure 2 is devoted to $d E / d x$ quantities, in atomic units, obtained for $\mathrm{Al}\left(r_{s} \simeq 2.13\right.$ ) with helium projectiles. The velocity range, in atomic units, is $v \in[0,0.6]$. The green dotted

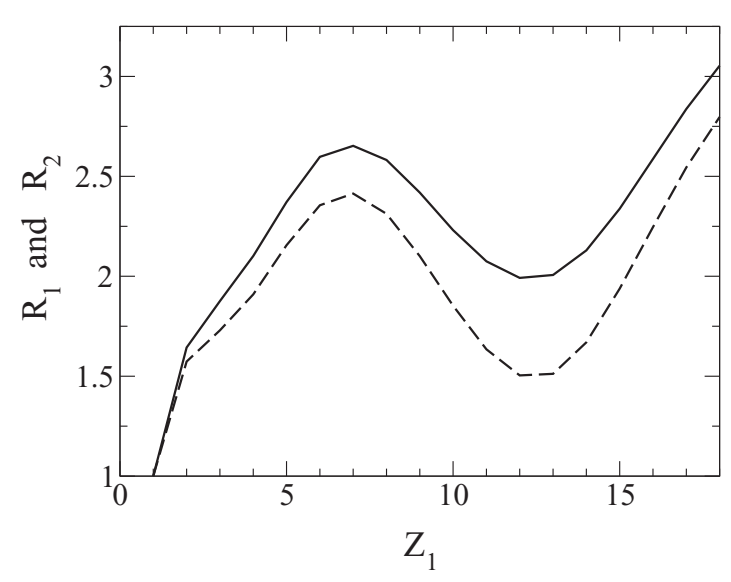

FIG. 1. Illustrative dimensionless ratios $R_{1}$ (dashed curve) and $R_{2}$ (solid curve), defined in the text, as a function of $Z_{1}$. The density parameter is $r_{s}=1.5$, which corresponds to a high-density degenerate electron gas, which would refer to the plasma frequency of Au.

curve represents a simple (with $Z_{1}=2$ ) linear-response (firstorder Born) approximation, where the electron gas dielectric function at the random-phase approximation (RPA) level, i.e., without static or dynamic local-field corrections [30], is used. In such an approximate theory, the stopping is proportional to $Z_{1}^{2}$. The corresponding form, employed in a foundational work

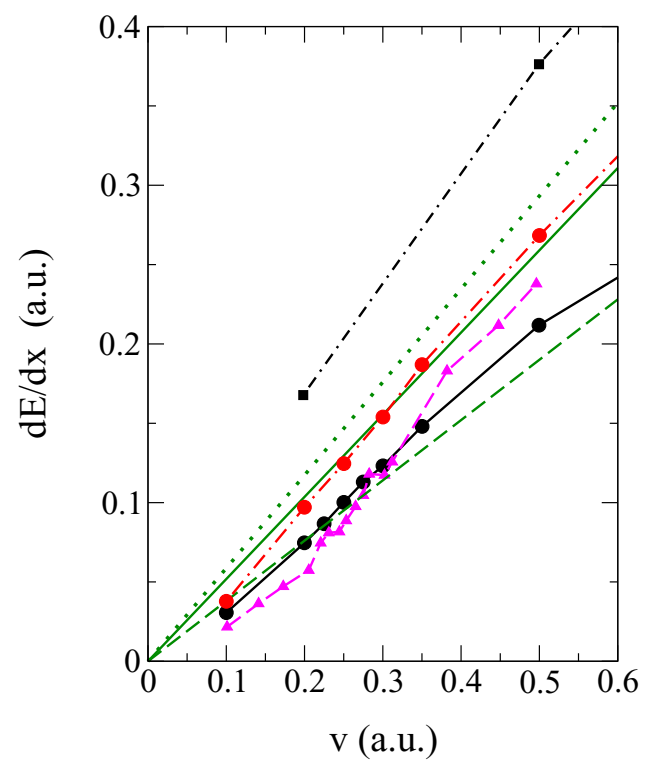

FIG. 2. Stopping power $d E / d x$ for helium projectiles as a function of the velocity $v \in[0,0.6]$. The present results are represented by the green solid and green dashed curves. Symbols are taken from Fig. 5(b) of [7] (black solid and red dash-dotted curves with closed circles) and from Fig. 5(b) of [24] (black squares). Data for Al are plotted by a dashed magenta curve with closed triangles. Their systematic and statistical errors are analyzed in the experimental work [15]. Finally, the green dotted curve shows Eq. (10) with $Z_{1}=2$. See the text for further details. 
[19] as well, is given by

$$
\left(\frac{d E}{d x}\right)_{R P A}=v \frac{4}{3 \pi}\left\{\frac{Z_{1}^{2}}{2}\left[\ln \left(a+\frac{2}{3}\right)-\frac{3 a-1}{3 a+2}\right]\left(\frac{3 a}{3 a-1}\right)^{2}\right\},
$$

where the abbreviation $a=\pi v_{F}$ is used. Its value is about $a \simeq 2.83$ for Al. This expression is a particular realization of the model discussed in the Introduction with $\sigma_{\mathrm{tr}}\left(v_{r}\right) \propto 1 / v_{r}^{4}$.

Our present results (at $Z_{1}=2$ ) correspond to the green solid curve $\left(Q^{(1)}+Q^{(2)}\right)$ and the green dashed curve $\left(Q^{(1)}\right)$. The black solid and red dash-dotted curves with closed circles are taken from Fig. 5(b) of [7]. They refer to off-channeling and channeling conditions, respectively. Notice that an earlier TDDFT result of [6] (not shown here) agrees precisely with the black curve. There is a fortuitous similarity between the RPA result for the homogeneous electron gas and results plotted via green solid and red dash-dotted curves with closed circles. Neither the screening treatment nor the scattering description of RPA is correct for a nonlinear situation. For protons, where reasonable agreement $[15,16,21]$ between nonlinear $Q^{(1)}\left(v_{F}\right)$ and data was found, Eq. (10) with $Z_{1}=1$ would give a serious underestimation [19].

Experimental [15] data, used already in TDDFT for comparison [7], are plotted here with a magenta dashed curve with closed triangles. This curve signals a two-slope behavior with linearities in the projectile velocity. Remarkably, a quite similar, i.e., two-slope, behavior was found in [16] for $\mathrm{Ni}$ $\left(r_{s} \simeq 1.8\right)$ with a singly ionized $\mathrm{He}^{+}$intruder. There a comparison with TDDFT results [31] was made by using 1.15 as a multiplying factor for the simulation-based results. As we already discussed above, we can image such a two-slope behavior within the present theoretical framework with certain, presumably closest-approach-dependent [32], finer-tuning of our two nonlinear channels. A complete convergence is still not achieved. The two black squares, for the $\alpha$ projectile, are taken from Fig. 5(b) of [24], for our velocity range. They are based on TDDFT with an optimized, localized atomistic, Gaussian basis set. We speculate that, for extended systems with slow ions, the screening action of the metallic electron gas needs further consideration. Moreover, a singly ionized $\mathrm{He}^{+}$intruder, instead of $\mathrm{He}^{2+}$, might be closer to the experimental situation.

In Fig. 3 we plot the observable quantities $(1 / v)(d E / d x)$ as a function of $Z_{1}$. The experimental data (black circles and triangles) were used earlier [19] for a comparison with $Q^{(1)}$, which is denoted here by a dashed curve. It was stated in that pioneering work that there is substantial disagreement with data in magnitude, particularly around the minimum. Our result $Q^{(1)}+Q^{(2)}$ is denoted by a solid curve. Notice that data symbols, without error bars, refer to $v=0.411$ (circles) and $v=0.826$ (triangles). The target is the frequently used prototype of free-electron metal, aluminum. By inspection, one can observe an essential improvement in agreement between the data and our approach. Here we return to the experiment in [26], i.e., to the above-mentioned surface experiment. There, although with somewhat smaller deviations from the conventional $Q^{(1)}\left[v_{F}(z)\right]$-type scaling, also a systematic upward enhancement in stopping power was established. In the present two-channel modeling, such an enhancement can be associated with a $Q^{(2)}$-proportional contribution.

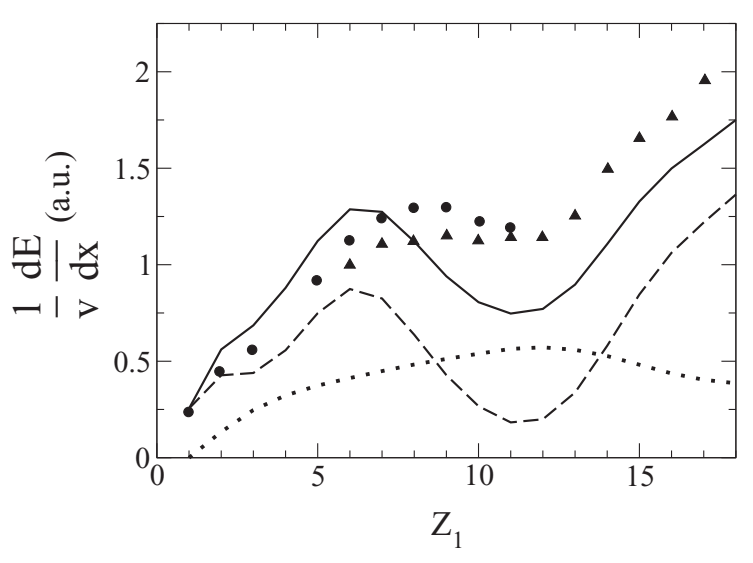

FIG. 3. Results for the frictionlike coefficients $(1 / v)(d E / d x)$ are plotted in atomic units. The dashed and dotted curves show Eqs. (8) and (9), respectively. The sum of Eqs. (8) and (9), the solid curve, refers to our approach. The experimental data points (for $\mathrm{Al}$ ) are the same, which were used in a pioneering work by Echenique et al. [19]. They are denoted by closed circles and triangles.

\section{CONCLUSION}

In this theoretical paper we have investigated the problem of an average retarding force of metallic targets for slow projectiles. Beyond the well-known contribution, established for electron-slow-intruder scattering in a degenerate electron gas, a contribution was derived which is associated with chargechange cycles due to lattice ions. Our main closed result was given by Eq. (6), which is, in the terminology of this subfield of physics, a nonlinear form, similar to the more conventional one in Eq. (2). These forms were implemented here by standard phase shifts obtained by applying the orbital-based DFT to screening in an electron gas. For helium projectiles, we made comparisons with selected results of experiments [15] and large-scale simulations [6,7] in TDDFT for a freeelectron-like metal, Al. With our contribution to the retarding force, the agreement with these results is improved.

As we discussed in Sec. I, our channel describes a sudden perturbation which is not explicit in recent TDDFT simulations. There a smoothed evolving picture was employed, without fast local charge-changing processes. As Fig. 2 indicated, our green curves bracket the TDDFT outputs obtained for channeling and off-channeling conditions within an evolving picture. We believe that further efforts in TDDFT are needed to tackle explicitly charge-changing processes. However, by construction, TDDFT simulations are able to model the lattice-related details of realistic targets. In order to get further information on the capabilities of different theoretical methods, the problem of projectiles in alloys could be an important one. There different lattice ions could influence (presumably due to different closest approaches) the chargechanging fast processes, giving an opportunity to see the advantage of our modeling over those based on a smoothed picture in the time domain.

A challenging problem in ratio-data interpretation [14] for $\mathrm{Mg}$ was discussed as well. Our two-channel-based result was in reasonable agreement with data at around $v=v_{F} \simeq 0.7$. The conventional theoretical estimation was not in agreement 
with experiment. A detailed comparison with data for $Z_{1} \in$ $[1,18]$ in $\mathrm{Al}$ was made and improved agreement was found. Based on these agreements, we suggest further efforts within TDDFT along these lines. The percentage differences (about $400 \%$ and $40 \%$ ) of the conventional $Q^{(1)}$ (dashed curve) and the $Q^{(1)}+Q^{(2)}$ (solid curve) results in Fig. 3 for $Z_{1}=12$ in comparison with experimental data on pure Al target heralds that alloy targets could be relevant candidates to understand differences between the theoretical attempts discussed in this work.

Note that a recent adiabatic modeling [29], motivated by experiment in [21], also results in remarkable deviations from a simple modeling with $Q^{(1)}\left(v_{F}\right)$. There the density inhomogeneity was considered, via lattice-atom-volume averaging of $Q^{(1)}\left[v_{F}(r)\right]$, as a modulating effect. Such an averaging was applied successfully [33] for stopping of swift $Z_{1}= \pm 1$ in order to discuss the charge-sign effect in Si. In the theoretical modeling [29] a remarkable similarity to experimental effective charges (defined in Fig. 1) was obtained in such a way. Furthermore, it was suggested that calculations within largescale TDDFT simulations would be useful to demonstrate the strength of the underlying [29] approach. We share this suggestion for realistic TDDFT. The suggestion made above on another important challenge with $\mathrm{Mg}\left(r_{s} \simeq 2.7\right)$ or with $\mathrm{Ca}$ $\left(r_{s} \simeq 3\right)$ is in accord with this.

Thus, at this moment, we have two, i.e., $q$-dependent and inhomogeneity-dependent, effects which result in enhancement in the electronic stopping power beyond the conventional, i.e., $Q^{(1)}$-dependent, theoretical estimation. Both seem to be, a priori, relevant in reality. Their proper weights and interplay need further investigation. Cases with self-irradiated condition $[34,35]$ could be especially important in this $(q \neq 0)$ respect, for instance, $\mathrm{Ni}$ ions in a $\mathrm{Ni}$ target [35]. In such a symmetric case we can image (for a metal) even $q=2$ for our $Q^{(2)}$ channel. For $r_{s} \simeq 2$, the Ni ion with its $Z_{1}=28$ represents the second minimum in the $Z_{1}$ oscillation $[18,20]$. In our modeling we get $Q^{(1)}+Q^{(2)} \simeq 0.28+0.72 q^{2}$.

At $q=2$ and $v=1$, one arrives at $\left(Q^{(1)}+Q^{(2)}\right) / Q^{(1)} \simeq$ 11 ; thus the corresponding stopping power would change steeply to about $d E / d x \simeq 160 \mathrm{eV} / \mathrm{A}$. For transition metals, which show a high electronic stopping power [35], the spin-flip process needs a thorough investigation. The electron spin direction is no longer conserved during electron-atom collision. One has to consider the total angular momentum $\mathbf{j}=\mathbf{l}+\mathbf{s}$ operator in order to construct a complete set of spin-angle functions which are needed for expansions. We left this exciting subproblem in stopping theory with a new (spin) degree of freedom to future studies.

Note that at high ion velocity, our term would scale as $(q / v)^{2}$ with respect to the conventional, i.e., Bethe-like [1], leading one $[7,24]$. There a term with $[q(v) / v]^{2}$ can give a slowly vanishing enhancement. Thus the high-velocity limit, under self-irradiated condition [35], also requires further investigation. The precise relevance of permutation-based similarity-aided level crossing [22,36] behind higher- $q(v)$ values seems to be another interesting subproblem in stopping theory. The Bethe limit, especially for metals with their dense electron gas, is not a simple cumulative sum of isolated atomic contributions [37].

Based on the established capability of our modeling for metals, we believe that the two-channel approach developed here can find application in other important fields as well, for instance, in the friction problem of diatomic molecules during their dissociative adsorption on metallic surfaces. There, based on an empirically motivated local-density-friction approximation, a local $Q^{(1)}\left[v_{F}(r)\right]$ is employed [38]. We argue here that transient electronic processes, due to dissociation in an electron gas, could be related to $Q^{(2)}$ in Eq. (6). For instance, the case of $\mathrm{N}$, with its $Z_{1}=11$, might be a good candidate, as Table I suggests. We stress, however, that at high target temperatures, the coupling to phonon modes, i.e., to quanta of lattice vibrations, can open a new [39] channel to inelastic processes, beyond the frictionlike channel discussed in our present study for cold metals. Still, as Fig. 1 of [39] signals, the proper magnitude of this latter channel could be important. Indeed, the so-far neglected [39] charge-transfertype [related to $Q^{(2)}\left(v_{F}\right)$ ] processes, especially with highly reactive molecules, may have impact on conclusions.

We close with a few general comments. The wave functions of the conventional orbital-based DFT for embedded $Z_{1}$ were used [18-20] here to calculate the induced electron density. That is the basic variable of the underlying variational theory. The phase shifts are therefore auxiliary quantities [18]. Their sums over angular momentum quantum numbers always satisfy the associated neutrality condition of a self-consistent orbital-based approximation, i.e., the Friedel sum rule and the Levinson theorem [13] for local interactions. Since these are satisfied by construction for any form of a local many-body term in the Schrödinger-like equations, the physical quality of DFT results needs further, i.e., energetic, justification.

However, in accord with closely related statements [18,20], the highly improved quantitative agreement with experimental facts justifies, a posteriori, our phase-shift-based two-channel modeling with a different term for the retarding force. Generally, and in accord with the basic attempt of Landau and Lifshitz [36] for Fermi liquids, a modeling is good if it contains few adjustable elements, agrees with several observations, and makes controllable predictions. We stress, finally, that the truly exciting theoretical problem of interparticle interaction, i.e., correlated motion of electrons, is considered in stopping calculations only at the mean-field level. However, at least for a prototypal two-particle correlated model system, a recent exact result [40] for the energy shift in time-dependent (passing) perturbations indicates that proper independent modes, rather than effective single-particle states, could pave the way for future developments.

\section{ACKNOWLEDGMENTS}

One of us (I.N.) is indebted to P. M. Echenique for his continuous interest and advice. We are thankful to P. Bauer, R. Díez Muino, J. I. Juaristi, I. Maliyov, D. Primetzhofer, and D. Sánchez-Portal for very useful discussions. This work was supported in part by the Spanish Ministry of Economy and Competitiveness (Project No. FIS2016-76617-P). 
[1] L. D. Landau and E. M. Lifshitz, Quantum Mechanics (Pergamon, London, 1958).

[2] P. Sigmund, Particle Penetration and Radiation Effects (Springer, Berlin, 2014).

[3] E. Bonderup, Lecture Notes on Penetration of Charged Particles through Matter, 2nd ed. (University of Aarhus, Aarhus, 1981).

[4] G. Zwicknagel, C. Toepffer, and P. G. Reinhard, Phys. Rep. 309, 117 (1999).

[5] I. Nagy and B. Apagyi, Adv. Quantum Chem. 46, 267 (2004), and references therein..

[6] M. A. Zeb, J. Kohanoff, D. Sánchez-Portal, and E. Artacho, Nucl. Instrum. Methods Phys. Res. Sect. B 303, 59 (2013), and references therein.

[7] A. Schleife, Y. Kanai, and A. A. Correa, Phys. Rev. B 91, 014306 (2015).

[8] A. Salin, A. Arnau, P. M. Echenique, and E. Zaremba, Phys. Rev. B 59, 2537 (1999).

[9] B. A. Lippmann, Phys. Rev. Lett. 15, 11 (1965).

[10] G. D. Gaspari and B. Györffy, Phys. Rev. Lett. 28, 801 (1972).

[11] L. Bönig and K. Schönhammer, Phys. Rev. B 39, 7413 (1989).

[12] N. H. March and G. G. N. Angilella, Exactly Solvable Models in Many-body Theory (World Scientific, Singapore, 2016), and references therein.

[13] P. G. Burke, Potential Scattering in Atomic Physics (Plenum, New York, 1977).

[14] M. Bergsmann, P. Hörlsberger, F. Kastner, and P. Bauer, Phys. Rev. B 58, 5139 (1998).

[15] D. Primetzhofer, S. Rund, D. Roth, D. Goebl, and P. Bauer, Phys. Rev. Lett. 107, 163201 (2011), and references therein.

[16] T. T. Tran, L. Jablonka, B. Bruckner, S. Rund, D. Roth, M. A. Sortica, P. Bauer, Z. Zhang, and D. Primetzhofer, Phys. Rev. A 100, 032705 (2019), and references therein.

[17] A. Arnau, M. Penalba, P. Echenique, and F. Flores, Nucl. Instrum. Methods Phys. Res. Sect. B 69, 102 (1992).

[18] M. J. Puska and R. M. Nieminen, Phys. Rev. B 27, 6121 (1983).

[19] P. M. Echenique, R. M. Nieminen, J. C. Ashley, and R. H. Ritchie, Phys. Rev. A 33, 897 (1986), and references therein.

[20] I. Nagy, A. Arnau, and P. M. Echenique, Phys. Rev. A 40, 987 (1989).

[21] M. A. Sortica, V. Paneta, B. Bruckner, S. Lohmann, T. Nyberg, P. Bauer, and D. Primetzhofer, Sci. Rep. 9, 176 (2019).
[22] O. B. Firsov, Zh. Eksp. Teor. Fiz. 36, 1517 (1959) [Sov. Phys.JETP 36, 1076 (1959)], and references therein.

[23] F. Grüner, F. Bell, W. Assmann, and M. Schubert, Phys. Rev. Lett. 93, 213201 (2004).

[24] I. Maliyov, J. P. Crocombette, and F. Bruneval, Phys. Rev. B 101, 035136 (2020).

[25] P. Riccardi, R. A. Baragiola, and C. A. Dukes, Phys. Rev. B 92 , 045425 (2015).

[26] H. Winter, J. I. Juaristi, I. Nagy, A. Arnau, and P. M. Echenique, Phys. Rev. B 67, 245401 (2003), and references therein.

[27] T. A. Gomez, T. Nagayama, C. J. Fontes, D. P. Kilcrease, S. B. Hansen, M. C. Zammit, D. V. Fursa, A. S. Kadyrov, and I. Bray, Phys. Rev. Lett. 124, 055003 (2020).

[28] J. A. Frenje, R. Florido, R. Mancini, T. Nagayama, P. E. Grabowski, H. Rinderknecht, H. Sio, A. Zylstra, M. Gatu Johnson, C. K. Li, F. H. Seguin, R. D. Petrasso, V. Y. Glebov, and S. P. Regan, Phys. Rev. Lett. 122, 015002 (2019).

[29] F. Matias, P. L. Grande, M. Vos, P. Koval, N. E. Koval, and N. R. Arista, Phys. Rev. A 100, 030701(R) (2019).

[30] I. Nagy, J. László, and J. Giber, Z. Phys. A 321, 221 (1985).

[31] E. E. Quashie and A. A. Correa, Phys. Rev. B 98, 235122 (2018).

[32] I. Nagy, I. Aldazabal, and M. L. Glasser, J. Phys. B 45, 095701 (2012).

[33] I. Nagy, Phys. Rev. A 65, 014901 (2001), and references therein.

[34] A. Lim, W. M. C. Foulkes, A. P. Horsfield, D. R. Mason, A. Schleife, E. W. Draeger, and A. A. Correa, Phys. Rev. Lett. 116, 043201 (2016).

[35] R. Ullah, E. Artacho, and A. A. Correa, Phys. Rev. Lett. 121, 116401 (2018).

[36] A. B. Migdal, Qualitative Methods in Quantum Theory (Benjamin, London, 1977).

[37] J. A. Nobel, S. B. Trickey, J. R. Sabin, and J. Oddershede, Chem. Phys. 309, 89 (2005).

[38] M. Alducin, R. Díez Muino, and J. I. Juaristi, Prog. Surf. Phys. 92, 317 (2017).

[39] L. Martin-Gondre, M. Alducin, G. A. Bocan, R. Díez Muino, and J. I. Juaristi, Phys. Rev. Lett. 108, 096101 (2012), and references therein.

[40] I. Nagy and I. Aldazabal, Adv. Quantum Chem. 80, 23 (2019). 